\title{
Photospheric flows measured with TRACE
}

\author{
J. M. Krijger ${ }^{1}$, T. Roudier ${ }^{2}$, and M. Rieutord ${ }^{2}$ \\ 1 Sterrekundig Instituut, Postbus 80000,3508 TA Utrecht, The Netherlands \\ e-mail: J.M.Krijger@astro.uu.nl \\ ${ }^{2}$ Laboratoire d'Astrophysique de Toulouse, Observatoire Midi-Pyrénées, 14 avenue E. Belin, 31400 Toulouse, \\ France \\ e-mail: roudier@bagn.obs-mip.fr; rieutord@obs-mip.fr
}

Received 16 January 2002 / Accepted 14 March 2002

\begin{abstract}
We analyse white-light image sequences taken with the Transition Region and Coronal Explorer (TRACE) using an optimised local correlation tracking (LCT) method to measure the horizontal flows in the quiet solar photosphere with high spatial $(1 \mathrm{arcsec})$ and temporal (5 min) resolution. Simultaneously taken nearultraviolet images from TRACE confirm that our LCT-determined flows recover the actual supergranulation pattern, thus proving that the topology of the horizontal flow distribution and network assembly may be studied from long-duration TRACE white-light sequences with our method.
\end{abstract}

Key words. Sun: photosphere - Sun: chromosphere - Sun: granulation

\section{Introduction}

Convective flows dominate the stucture of and the energy transport in the outer parts of the Sun up to the deep photosphere. Understanding the convective flow and its interaction with the magnetic fields is important since convective motions are believed to drive many important processes on the sun, e.g., (i) - the solar dynamo through interaction with solar rotation, (ii) - the $p$-mode oscillations that are used to probe the solar interior, (iii) magneto-hydrodynamic waves which supply energy to the chromosphere, and (iv) - foot-point motions that lead to magnetic reconnection which may heat the corona.

The most visible product of convection is the solar granulation with a characteristic scale of $1000 \mathrm{~km}$, lifetime of about 10 min (e.g., Bray et al. 1984), and easily recorded in broadband images. The solar granulation is reproduced remarkably well in numerical simulations (e.g., Stein \& Nordlund 1998). The larger granulation scales, meso- and supergranulation, are less understood. The existence of convection at the mesogranular scale is still in debate (e.g., Rieutord et al. 2000; Ploner et al. 2000), while study of the supergranular pattern is difficult because it does not show directly in white light whereas the magnetic network and the chromospheric brightness network map supergranular cell boundaries only incompletely. The new technique of time-distance helioseismology (Duvall et al. 1993) has become very popular for studying the

Send offprint requests to: J. M. Krijger,

e-mail: J.M.Krijger@astro.uu.nl supergranulation, but results in controversal findings (e.g., Hathaway et al. 2002) which require further investigation. The supergranule scales are still debated (see Beck \& Duvall 2001), with the current estimates for the length scale between $13 \mathrm{Mm}$ and $35 \mathrm{Mm}$ (e.g., Hagenaar et al. 1997) and a lifetime between 15 hours and 52 hours depending on scale (e.g., Wang \& Zirin 1989; Simon et al. 1995).

As shown by Rieutord et al. (2001) the flow speeds determined from brightness changes describing granular motions are an indication of the horizontal motion of the associated matter. Tracking this granular structure enables determination of the longer-lived and wider horizontal flows (November \& Simon 1988) and one may so study the assembly and topological evolution of the magnetic network at these scales.

Different attempts (e.g., Leighton et al. 1962; Brandt et al. 1994; 1999, 2000) have been made to determine quiet-sun surface flows from ground-based observations. These observations were limited by small field of view (FOV), short duration, and the presence of seeing which hampers precise flow evaluation. Seeing is best avoided by putting telescopes in orbit. The SOUP instrument onboard Spacelab 2 collected a sequence of too short a duration (28 min) to study supergranular $(>15 \mathrm{hrs})$ pattern changes (Simon et al. 1988). The MDI instrument on-board the SOHO satellite can observe for much longer duration but is limited by the fixed pointing of its high resolution field. The line-of-sight velocity images obtained 
by MDI are much used with time-distance helioseismology (e.g., De Rosa et al. 2000; 2002).

Image sequences from the TRACE satellite (Handy et al. 1999) do not suffer from seeing and have a somewhat better resolution (1 arcsec) than MDI (1.3 arcsec). TRACE offers a large FOV $\left(512 \times 512 \operatorname{arcsec}^{2}\right)$ and long duration observations (up to a week) by following a given area on the solar surface by correcting for solar rotation. Also, TRACE permits quasi-simultaneous observations in both white light (low photosphere) and near-ultraviolet (upper photosphere) wavelengths. The bright chromospheric network visible in the near-ultraviolet wavelengths can be used to show the location of the magnetic network, which is governed by the flows, in the absence of magnetograms allowing a direct check. However, so far it was not clear whether the TRACE resolution of 1 arcsec is good enough to obtain reliable flow estimates since it resolves the granulation which is to be used as flow tracer only partially.

In this paper we test whether it is possible to use the TRACE white light observations despite their low resolution for measuring horizontal velocity fields, by optimising the LCT method of November \& Simon (1988) and using "isotropic divergence" (Roudier et al. 1999; Rieutord et al. 2000). The (dis)advantages of the LCT and other tracking techniques used by different authors are presented and discussed in Roudier et al. (1999). For a discussion of previous studies using feature tracking, see Rieutord et al. (2000). Our test proves that our modified LCT method with optimised parameters and data reduction can be used to derive horizontal flows from longer TRACE white-light image sequences.

The organisation of the paper is as follows. The observations and reductions are presented in the next section. In Sect. 3 we display the results of the analysis with the conclusions following in Sect. 4.

\section{Observations and reduction}

\subsection{TRACE sequence}

We use a TRACE image sequence taken on October 14, 1998 between 8:12-12:00 UT. TRACE observed a very quiet rectangular area of the solar surface at disk center (see Fig. 1) in three ultraviolet passbands (centered at $\lambda=$ $1700 \AA, 1600 \AA$, and $1550 \AA$ ) and a broadband white-light passband. A nominal cadence of $21 \mathrm{~s}$ for each individual passband (but longer occasionally) was used. Only $512 \times$ 512 pixel $^{2}$ of the $1024 \times 1024$ pixel $^{2}$ TRACE CCD camera with 0.5 arcsec pixels ( $366 \mathrm{~km}$ on the Sun) were read out due to telemetry limitations. The satellite pointed at a fixed location on the apparent solar disk without following solar rotation during the observation. The observations are specified in Table 1 . Figure 2 shows as example pair of white-light and $1700 \AA$ images.

In this analysis only the lower half of the observed field $\left(256 \times 128 \operatorname{arcsec}^{2}\right)$ was used, due to computer resource restrictions. The images were reduced following standard

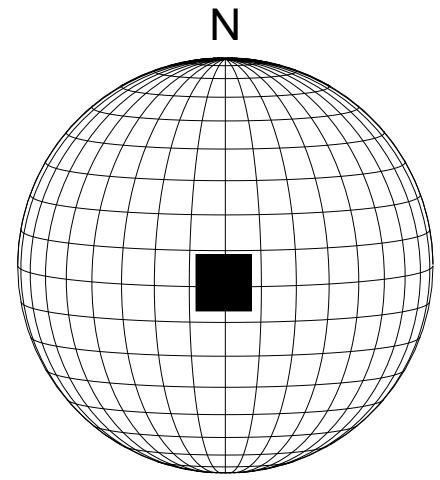

Fig. 1. Solar location of the field observed with TRACE on October 14, 1998, slightly off disk center. Only the lower half of this field was used for the analysis presented in this paper. The grid shows heliocentric longitude and latitude.

procedures $^{1}$ (dark current subtraction, flat field correction). The sequences were subsequently aligned to remove solar rotation and satellite jitter using cross-correlation aligning the ultraviolet images to other images of the same passband and to the other passbands within a few tenths of a pixel. Differential rotation across the field during the entire sequence had only a small effect ( 0.4 pixel) and was neglected. Due to solar rotation and satellite jitter only part of the FOV was fully sampled $\left(452 \times 246\right.$ pixel $^{2}$, $226 \times 123 \operatorname{arcsec}^{2}$ or $\left.165 \times 90 \mathrm{Mm}^{2}\right)$. More details on these data and its reduction are given in Krijger et al. (2001). More information on reducing TRACE data is available in the TRACE Analysis Guide ${ }^{2}$.

Table 1. TRACE observations used in this paper.

\begin{tabular}{lc}
\hline \hline date & October 14, 1998 \\
program sequence & TDT.any_frames \\
image size [pixel] & $512 \times 512$ \\
image size [arcsec] & $256 \times 256$ \\
used image size [pixel] & $452 \times 246$ \\
used image size [arcsec] & $226 \times 123$ \\
size pixel [arcsec] & 0.5 \\
size pixel [km] & 366 \\
number of images & 611 per passband \\
$X_{\text {cen }}, Y_{\text {cen }[\operatorname{arcsec}]}$ & $-13,-82$ \\
$L_{\text {cen }}, B_{\text {cen }}[\mathrm{deg}]$ & $-0.77,1.04$ \\
duration [UT] & $08: 12-12: 00$ \\
cadence [s] & 21.8625 \\
exposure $1700 \AA$ [s] & 2.0480 \\
exposure $1600 \AA$ [s] & 1.0239 \\
exposure $1550 \AA$ [s] & 9.7400 \\
exposure white light [s] & 0.0064 \\
\hline
\end{tabular}

\subsection{Local correlation tracking}

In order to take out the five-minute oscillation, all horizontal movements faster then $4 \mathrm{~km} \mathrm{~s}^{-1}$ were removed from the

\footnotetext{
${ }^{1}$ http://www.lmsal.com/solarsoft/

2 http://diapason.lmsal.com/ bentley/guides/tag/
} 


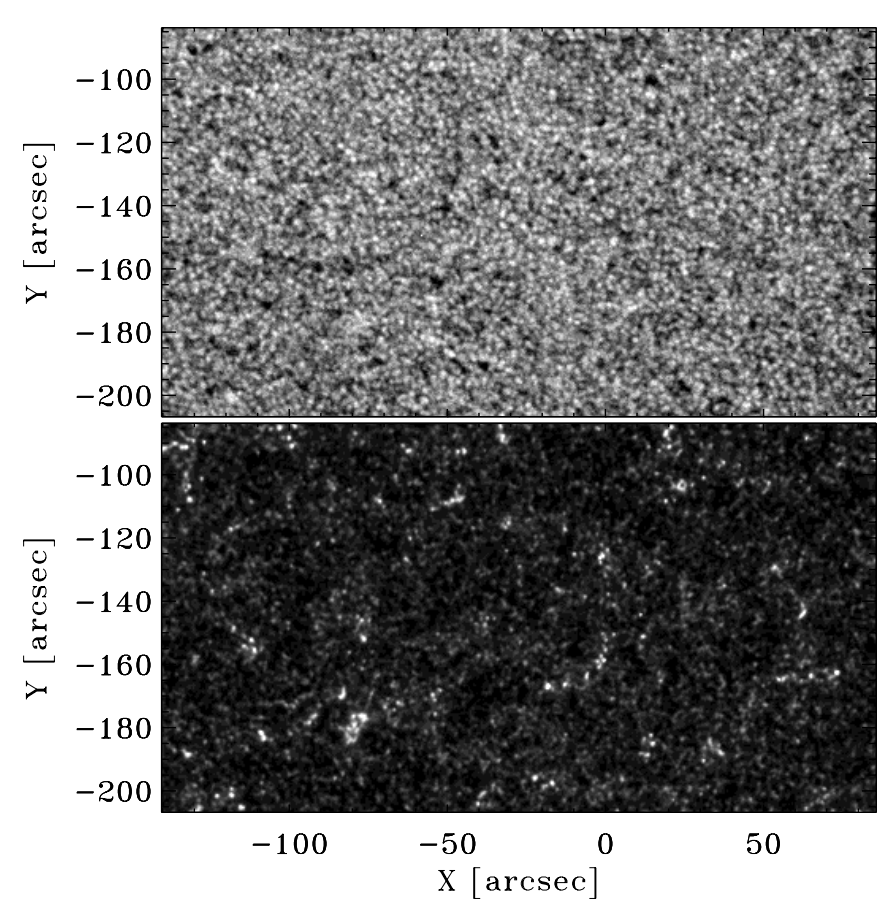

Fig. 2. Top: sample white-light image taken by TRACE at 10:03 UT, Oct. 14 1998. With a resolution of only 1 arcsec TRACE only resolves the larger granules. Smaller granules can jointly appear erroneously as large granules when close enough. No large-scale or magnetic structures are discernable. Bottom: corresponding $1700 \AA$ image taken by TRACE 2 s earlier. The brightest features belong to the chromospheric network. The latter stands out more clearly in the time-average display at bottom-left in Fig. 6. Axes: $X$ and $Y$ in arcsec from disk center.

white-light sequence by $3 \mathrm{D}$ Fourier filtering (Title et al. 1989).

Subsequently, each individual white-light image was enlarged by a factor of 4 (using interpolation) in order to increase the number of points used in subsequent correlation computations since the noise in the velocity measurements is proportional to $1 / \sqrt{N}$ where $N$ is the number of points used for the correlation. A test on blurred high resolution ground-based observations showed that this enlargement allows better recovery of the actual velocity fields.

The method used below is described in more detail in Roudier et al. (1999). After enlargement the images were filtered using a $1 \operatorname{arcsec}^{2}$ Gaussian window. Tests showed that a larger window samples multiple granules and measures only their group-averaged motion, while a smaller window measures details below the resolution of TRACE (1 arcsec) and generates meaningless results. Subsequently, the images were transformed into a binary map of granules, using local curvature of the intensity pattern to identify granules following Strous (1995).

Horizontal velocity fields were calculated using these binarised maps with the LCT method of November \& Simon (1988) with $5 \mathrm{~min}$ resolution. Using LCT on binarised data was named the $\mathrm{LCT}_{\text {bin }}$ method by Roudier et al. (1999). For each 5 min temporal window

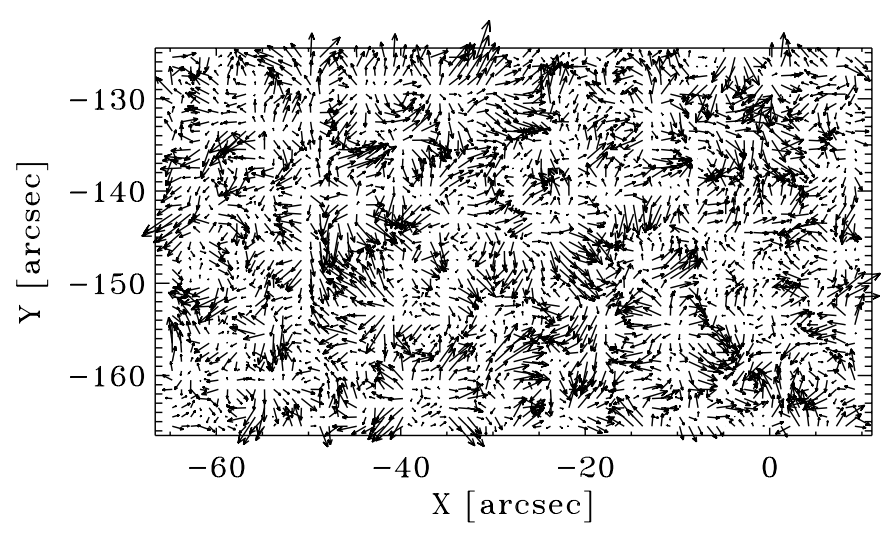

Fig. 3. Horizontal flows in a partial cutout from the measured horizontal velocity field at 1 arcsec resolution between 10:1010:15 October 14 1998. Each arrow shows the direction and magnitude of the velocity at a particular point with 1 arcsec length corresponding to $0.25 \mathrm{~km} \mathrm{~s}^{-1}$. Axes: $X$ and $Y$ in $\operatorname{arcsec}$ from disk center.

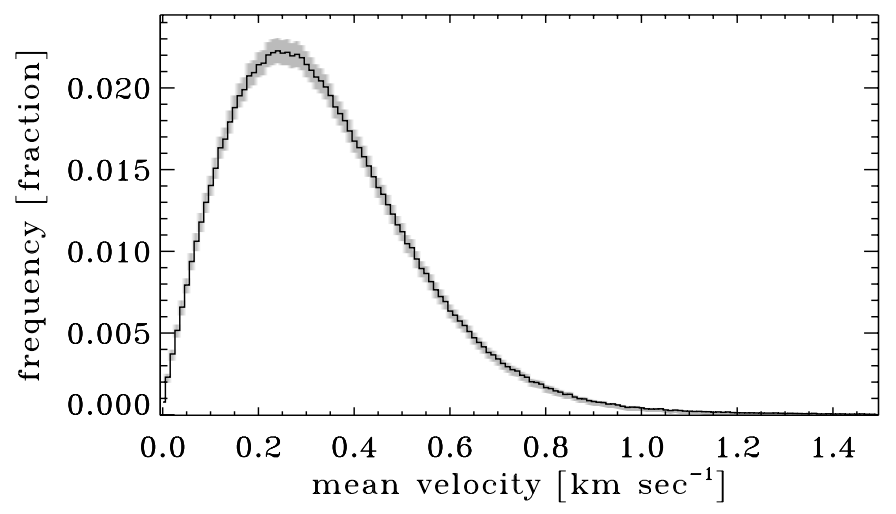

Fig. 4. Histogram of all measured mean horizontal velocities (both in $x$ and $y$ direction), with $3 \sigma$ estimates (shaded contours). Most velocities are below $1 \mathrm{~km} \mathrm{~s}^{-1}$, with a maximum around $0.25 \mathrm{~km} \mathrm{~s}^{-1}$.

15 sequential images were used. A test showed that using an artificial cadence of $\sim 1$ min (3 images per temporal window) instead of $21 \mathrm{sec}$ yields similar results. The period of $5 \mathrm{~min}$ was used as a compromise between the (mean) lifetime of the granules and noise in the resulting flow maps.

Figure 3 shows a sample result. Large-scale $(\sim 7 \mathrm{Mm})$ diverging and converging areas are visible as a mesoscale pattern. The statistical distribution of the measured flow speeds is shown in Fig. 4. The lower cut-off is due to the angular resolution set by the TRACE CCD pixel size. Most mean velocities are below $1 \mathrm{~km} \mathrm{~s}^{-1}$. This distribution is shifted towards the lower velocities compared to the granular mean velocity found in Roudier et al. (1999), due to the tendency of the $\mathrm{LCT}_{\text {bin }}$ method of smoothing out the smaller and faster patches in the velocity fields.

After the computation of successive mean-flow maps covering the whole sequence duration, one can insert artificial tracers or "corks" that follow horizontal motions over a longer lifetime than that of individual granules, and so mapping longer-lived flows (Simon et al. 1988). Figure 5 shows the final position of such corks, inserted at the start 


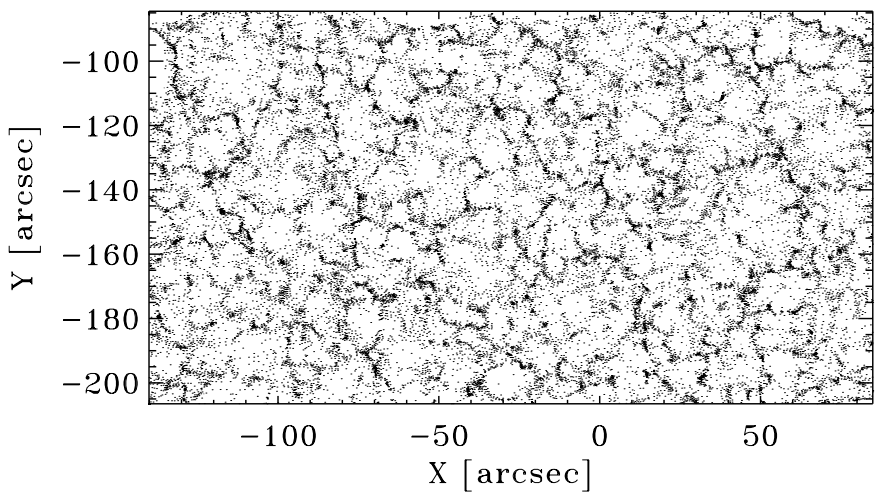

Fig. 5. This plot shows the final positions of about 30000 "corks" or tracers after 4 hours, that were initially distributed regularly and allow to freely flow on the velocity fields. Some tracers have not yet reached their final destination, but large scale structures are visible. Axes: $X$ and $Y$ in arcsec from disk center.

of the flow-map sequence. Large $(\sim 22 \mathrm{Mm})$ cell-like closed structures are visible, filled with smaller $(\sim 7 \mathrm{Mm})$ cells that are less well outlined. The large cells correspond to the supergranular network as we demonstrate in Figs. 6 and 7 . The smaller structures result at least partially from the short sequence duration by showing corks that have not yet reached their final supergranular-cell boundary destination.

\subsection{Isotropic divergence}

A quantity that can be derived from flow patterns is the horizontal divergence $\nabla \cdot v_{x y}$ which furnishes another presentation of the surface flows. Our interest is into sources of divergence/convergence, those locations where matter presumably appears at the surface by flowing upward (divergence) and where matter presumably disappears from the surface by flowing downwards (convergence), respectively. However, using the classical divergence calculation, one also finds a high divergence or convergence value at a particular location when the surrounding area as a whole is horizontally accelerating or decelerating. We therefore employ the "isotropic divergence" instead (Rieutord et al. 2000). We briefly describe both methods here.

Calculating the divergence at a certain point in a regular grid is a straightforward procedure by summing the difference in velocity (in orthogonal directions) of the 4 adjacent points. The mathematical definition for the classic divergence at location $(x, y)$ in a regular discrete grid is:

$\frac{v_{x}(x+1, y)-v_{x}(x-1, y)+v_{y}(x, y+1)-v_{y}(x, y-1)}{2 W}$,

with $W$ the distance between two gridpoints and $v_{x}, v_{y}$ the respective velocity components. The averaged horizontal divergence over the entire duration of the observation is shown in the top-left panel of Fig. 6.

The isotropic divergence is defined in each point as the sum of the difference (normalised by the distance) of the projected vector components of the velocity on the axis of the symmetrical points relative to the central one, in all the directions and computed for the two gridpoint-rings around this central point ( 8 points for the first ring and 16 points for the second ring). The mathematical definition for the isotropic divergence in location $(x, y)$ is:

$$
\sum_{s_{x}=-2}^{2} \sum_{s_{y}=-2}^{2} \frac{s_{x} v_{x}\left(x+s_{x}, y+s_{y}\right)+s_{y} v_{y}\left(x+s_{x}, y+s_{y}\right)}{2 W\left(s_{x}^{2}+s_{y}^{2}\right)}
$$

with $s_{x}, s_{y}$ the gridpoint distance from $x, y$ in their respective directions, which are not allowed to be zero at the same time (i.e., the velocity in the central point is not used for the divergence calculations). The resulting amplitudes peak at isotropic divergence/convergence sites such as exploding granules and sink holes. The averaged horizontal isotropic divergence is shown in the middle-left panel of Fig. 6.

\section{Results}

\subsection{Divergence}

Both the classic and isotropic divergences were evaluated and subsequently averaged over the total duration of the observation (8:12-12:00 UT). The results are shown in the two upper panels in the lefthand column of Fig. 6 . Using isotropic divergence yields a coarser pattern with a clearer indication of cellular structure at supergranular scale which is outlined by darker-than-average boundary segments.

\subsection{Chromospheric network}

The capacity of TRACE to simultaneously observe in the ultraviolet and the white-light allows us to compare the cork collection sites in Fig. 5 and the coarse grid-pattern of convergence sites in Fig. 6 to the actual structure of the chromospheric network. The pertinent ultraviolet observations from TRACE are described in more detail in Krijger et al. (2001). The TRACE $1600 \AA$ and $1700 \AA$ passbands show very similar features as $\mathrm{CaII} \mathrm{K}_{2 \mathrm{~V}}$ movies from ground-based telescopes (Rutten et al. 1999). The bottomleft panel of Fig. 6 shows the temporal average of the entire $1700 \AA$ sequence. The chromospheric network stands out much clearer in this time-average display than in the snapshot image in the lower panel of Fig. 2 because the intermittent internetwork features (mostly due to the chromospheric three-minute oscillation) average away while the more persistent network features are enhanced.

\subsection{Spatial comparison}

Comparison of Fig. 6 with Fig. 5 shows that the regions with highest cork density (Fig. 5) correspond closely to the bright ultraviolet network (bottom-left panel of Fig. 6). Similarly, comparing the temporally averaged divergences with the $1700 \AA$ temporal average image (lefthand panels of Fig. 6) indicates spatial correspondence between 

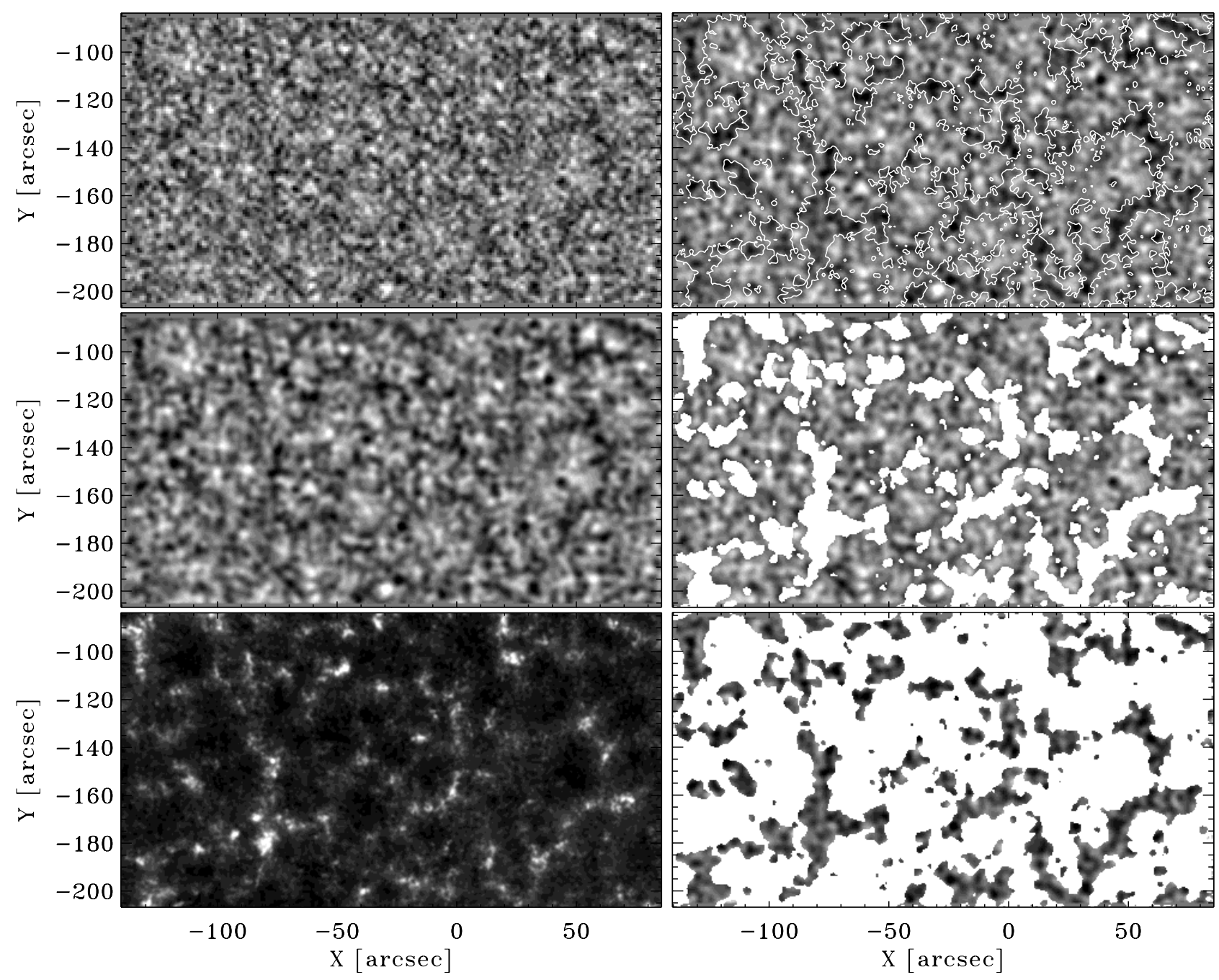

Fig. 6. Top left: classic divergence defined by Eq. (1) averaged over the entire duration (228 min) of the observation. Bright areas indicate locations where the flows are diverging, dark areas indicate converging flows. Middle left: same as first panel, but showing isotropic divergence as defined by Eq. (2). Large scale structures become more distinct. Bottom left: the average of the $1700 \AA$ sequence taken at the same time and location. The greyscale is logarithmic in order to bridge the large contrast. Top right: again showing the time-averaged isotropic divergence but now overlaid with the contours of the bright network from the bottom-left panel. A clear correlation between the dark converging areas and the bright network can be seen. Middle and bottom right: split of the top-right panel into internetwork-only and network-only displays, using a mask defined by the network contours. The split produces yet clearer visualisation of the spatial correlation between the dark converging areas and the bright network. Axes: $X$ and $Y$ in arcsec from disk center, all panels show the same subfield.

the (dark) converging areas and the bright chromospheric network. The righthand panels of Fig. 6 visualises this correlation by showing different comparisons between the isotropic divergence and the $1700 \AA$ image. In the topright panel the isotropic divergence is shown overplotted with the network contours of the $1700 \AA$ image. The two lower panels in the righthand column show the difference directly.

Finally, the spatial correlation between convergence sites and chromospheric network is illustrated in Fig. 7 by plotting $1700 \AA$ brightness versus the average divergence as a scatter diagram. It shows that most bright network features are located at convergence (negative divergence) locations. This proves that the surface flows which we have measured with our correlation tracking indeed represent long-term supergranular motions, even though Fig. 5 shows that not all corks have reached their final position in the supergranular cell borders.

\section{Conclusions}

We have demonstrated that, although TRACE resolution $(1 \mathrm{arcsec})$ is not good enough to fully resolve the solar granulation, it does suffice to determine the flow patterns over the solar surface that produce and control the topology of the solar network. Using the $\mathrm{LCT}_{\text {bin }}$ method high spatial (1 arcsec) and temporal (5 min) resolutions can be obtained. These resolutions have not been achieved before on seeing-free data with such a large FOV $\left(226 \times 123 \operatorname{arcsec}^{2}\right)$ and long duration $(228 \mathrm{~min})$. TRACE 


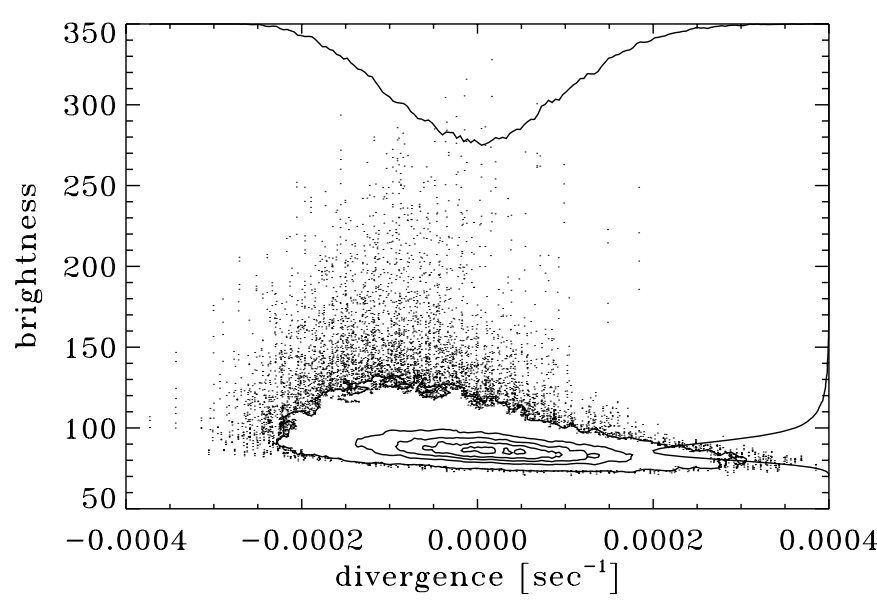

Fig. 7. Correlation between average $1700 \AA \AA$ brightness and average velocity field divergence shown as a pixel-by-pixel scatter plot, with density contours replacing individual points where they become too close. The curves along the axes measure the first moments per row and column, respectively. The locations with the brightest network (upward tail of the scatter cloud) correspond to negative divergence in the flow field.

allows a FOV up to $512 \times 512 \operatorname{arcsec}^{2}$ and observations centered at a given (co-rotating) solar location up to a week duration. Combination of such measurements with our method allows study of the distribution and network assembly over long time scales with longer TRACE WL sequences. These higher temporal and spatial resolutions allow a gain in the analysis of the signal (velocity) from high to low frequencies in the two domains. In particular, it helps to distinguish the smoothing effects of the data reduction and analysis from the solar flow fields at medium and low frequencies (spatial and temporal) and to learn about the influence of the smaller scales on to the largest ones. Our approach allows us to locate more precisely the largest diverging amplitude events of the flow field and to correlate their evolution to the UV bright points (oscillations, motions, etc.) which are located on the border of the supergranule.

Acknowledgements. J. M. Krijger's research is funded by The Netherlands Organization for Scientific Research (NWO). J. M. Krijger thanks the Observatoire Midi-Pyrénées for their hospitality and financial support during his visit there. We are indebted to R. J. Rutten for suggesting this research and many improvements to the manuscript and to referee J. G. Beck for his valuable comments.

\section{References}

Beck, J. G., \& Duvall, T. L. 2001, in Helio- and asteroseismology at the dawn of the millennium, ed. A. Wilson, Procs. of the SOHO 10/GONG 2000 Workshop (ESA SP-464), 577

Brandt, P. N., Rutten, R. J., Shine, R. A., \& Trujillo Bueno, J. 1994, in Solar Surface Magnetism, ed. R. J. Rutten, \& C. J. Schrijver, NATO ASI Series C 433 (Dordrecht: Kluwer), 251

Bray, R. J., Loughhead, R. E., \& Durrant, C. J. 1984, The solar granulation (2nd edition) (Cambridge University Press), 270

De Rosa, M., Duvall, T. L., \& Toomre, J. 2000, Sol. Phys., 192, 351

Duvall, T. L., Jefferies, S. M., Harvey, J. W., \& Pomerantz, M. A. 1993, Nature, 362, 430

Hagenaar, H. J., Schrijver, C. J., \& Title, A. M. 1997, ApJ, 481,988

Handy, B. N., Acton, L. W., Kankelborg, C. C., et al. 1999, Sol. Phys., 187, 229

Hathaway, D. H., Beck, J. G., Han, S., \& Raymond, J. 2002, Sol. Phys., 205, 25

Krijger, J. M., Rutten, R. J., Lites, B. W., et al. 2001, A\&A, 379, 1052

Leighton, R. B., Noyes, R. W., \& Simon, G. W. 1962, ApJ, 135,474

November, L. J., \& Simon, G. W. 1988, ApJ, 333, 427

Ploner, S. R. O., Solanki, S. K., \& Gadun, A. S. 2000, A\&A, 356,1050

Rieutord, M., Roudier, T., Ludwig, H.-G., Nordlund, Å., \& Stein, R. 2001, A\&A, 377, L14

Rieutord, M., Roudier, T., Malherbe, J. M., \& Rincon, F. 2000, A\&A, 357, 1063

Roudier, T., Rieutord, M., Malherbe, J. M., \& Vigneau, J. 1999, A\&A, 349, 301

Rutten, R. J., de Pontieu, B., \& Lites, B. W. 1999, in High Resolution Solar Physics: Theory, Observations, and Techniques, ed. T. R. Rimmele, K. S. Balasubramaniam, \& R. R. Radick, Procs. 19th NSO/Sacramento Peak Summer Workshop, ASP Conf. Ser., 183, 383

Simon, G. W., Title, A. M., Topka, K. P., et al. 1988, ApJ, 327,964

Simon, G. W., Title, A. M., \& Weiss, N. O. 1995, ApJ, 442, 886

Stein, R. F., \& Nordlund, A. 1998, ApJ, 499, 914

Strous, L. H. 1995, in Helioseismology, ed. J. Hoeksema, V. Domingo, B. Fleck, \& B. Battrick, Proc. of 4th Soho Workshop, ESA SP-376, 213

Title, A. M., Tarbell, T. D., Topka, K. P., et al. 1989, ApJ, 336,475

Wang, H., \& Zirin, H. 1989, Sol. Phys., 120, 1 